\title{
The European network for care of children with Paediatric Rheumatic Diseases: care across borders.
}

P. Dolezalova ${ }^{1}$, J. Anton ${ }^{2}$, T. Avcin ${ }^{3}$, M. Beresford ${ }^{4}$, P. A. Brogan ${ }^{5}$, T. Constantin ${ }^{6}$, Y. Egert ${ }^{7}$, I. Foeldvari $^{8}$, H.E. Foster ${ }^{9}$, V. Hentgen ${ }^{10}$, I. Kone-Paut ${ }^{11}$, J. Kummerle-Deschner ${ }^{12}$, P. Lahdenne ${ }^{13}$, B. Magnusson ${ }^{14}$, A. Martini ${ }^{15}$, L. McCann ${ }^{4}$, K. Minden ${ }^{16}$, S. Ozen ${ }^{17}$, C. Schoemaker ${ }^{18}$, P. Quartier $^{19}$, A. Ravelli ${ }^{15}$, I. Rumba ${ }^{20}$, N. Ruperto ${ }^{15}$, S. Vastert ${ }^{18}$, C. Wouters ${ }^{21}$, F. Zulian ${ }^{22}$, and N.M. Wulffraat ${ }^{18}$ for the SHARE consortium

1: Charles University in Prague and General University Hospital, Department of Paediatrics and Adolescent Medicine, Praha, Czech Republic

2: Hospital Sant Joan de Déu, University of Barcelona, Department of Pediatric Rheumatology, Barcelona, Spain

3: University Children's Hospital, University Medical Centre Ljubljana, Department of Allergology, Rheumatology and Clinical Immunology, Ljubljana, Slovenia

4: Alder Hey Children's NHS Foundation Trust, Paediatric Rheumatology, Liverpool, United Kingdom

5: University College London Great Ormond St Institute of Child Health. London, United Kingdom

6: Semmelweis University, Unit of Paediatric Rheumatology, Dept of Pediatrics, Budapest, Hungary

7: European network childhood arthritis patient organisation, Israel

8: Hamburg Centre for Pediatric and Adolescent Rheumatology, Hamburg, Germany

9: Newcastle Hospitals NHS Foundation Trust, Great North Children's Hospital, Newcastle Upon Tyne, United Kingdom

10: A. Mignot University Hospital Versailles - CEREMAI, Pediatric Rheumatology, Le Chesnay (Paris), France

11: Le Kremlin-Bicêtre University Hospital, Paris-Sud University, Paris, France

12: University Children's Hospital Tuebingen, Centre for Rheumatology / Autoimmune diseases, Tuebingen, Germany 
13: Children's Hospital, Helsinki University Central Hospital, Pediatric Rheumatology, Helsinki, Finland

14: Karolinska University Hospital, Pediatric Rheumatology Unit, Karolinska Hospital, Stockholm, Sweden

15: Giannini Gaslini Institute, Department of Pediatrics, Genoa, Italy

16: Charite University Hospital Berlin, Dpt. of Dermatology and Allergy, Berlin, Germany

17: Hacettepe University Children's Hospital, Department of Pediatric Nephrology and Rheumatology, Ankara, Turkey

18: Wilhelmina Kinderziekenhuis, Department of Pediatric Immunology and Rheumatology, University medical center Utrecht, Utrecht, Netherlands

19: Paris-Descartes University, Necker Children's Hospital, Paris, France

20: University of Latvia, Faculty of Medicine, Riga, Latvia

21: University Hospital Gasthuisberg, Department of Pediatrics, Division of Pediatric Rheumatology, Leuven, Belgium

22: Clinica Pediatrica I, Unità di Reumatologia Pediatrica, Padova, Italy 


\begin{abstract}
Objectives: To provide an overview of the paediatric rheumatology (PR) care in Europe to inform future specialist service provision.

Methods: An online survey was developed and presented to national coordinating centres of the Pediatric Rheumatology International Trials Organisation (PRINTO) representing a single EU member state (35 centres; Country survey); and to 288 individual PR centres (Centre and Disease Surveys) as a part of the EU project SHARE. The survey contained country and centre specific components covering organisation of PR care, composition of teams, education, healthcare and research facilities, and assessment of needs. The national coordinating centres completed both centre as well as country questions.
\end{abstract}

Results: Response rates were $83 \%$ for Country and $57 \%$ for Centre surveys. Data from both surveys were itemised to organisational, quality of care and educational aspects. Across the EU, only one paediatric rheumatologist is available per million population, located in one of the 288 centres with specialised PR care. In all EU member states, there is overall good access to specialist care and to approved medications, although off-label medication availability is worse in Eastern European countries. Full financial coverage is provided for most prescribed medications. PR education is widely available for physicians but is insufficient for allied health professionals. Participation in clinical trials is generally high. Among important gaps identified, lack of widely accepted clinical guidelines/recommendations; and insufficient adolescent transition management planning were highlighted.

Conclusions: This study provides a comprehensive analysis of specialist PR service provision across Europe. Seen from the perspective of health care providers, there are no major differences between EU member states. Rarity, chronicity and complexity of diseases form a major challenge to paediatric rheumatology care. Therefore, strengthening subspecialty networks (Paediatric Rheumatology European Society, PReS, PRINTO, and SHARE) and the recently created European Reference Networks (ERN) will facilitate provision an dissemination of standards of care and treatment recommendations to further improve patient-centred healthcare across Europe.

\title{
Introduction
}

The care and research for rare and complex disorders need strong collaborative networks, widely 
applicable treatment recommendations and standardized follow-up measures. We present an example of such a collaboration in the area of Paediatric Rheumatic Diseases (PRD), a heterogeneous group of rare inflammatory conditions affecting children. The EU project "Single Hub and Access point for paediatric Rheumatology in Europe" (SHARE, grant number No. 2011 1202) was set up to provide an overview of the current organisation of paediatric rheumatology (PR) with the aim to harmonise the care for children with rheumatic diseases in Europe (1). By doing so it promotes one of the main EU goals: to deliver equal health care across all EU member states. In this report we present results of a detailed survey from 35 European countries (online appendix), to provide an overview of paediatric rheumatology (PR) care in Europe to inform future specialist service provision, and identify currently unmet needs or deficiencies in specialist PR service provision.

\section{Methods}

The SHARE consortium included PR representatives from 15 European countries and 2 patient organisations (CS and YE). Participants had been actively involved in educational and scientific activities of the Paediatric Rheumatology European Society (PReS) and Pediatric Rheumatology International Trials Organisation (PRINTO). (2-7). The survey methodology and strategy were agreed at an initial consensus meeting. Three key components were identified and formulated into surveys probing Country, Centre and Disease-specific issues (the latter spanning all the main groups of PRD identified by consensus). The PRINTO provided a platform for online data capture. Access to the country survey was provided to one national PRINTO co-ordinator per country $(n=35)$, including Israel and Turkey. The latter two were included because of their long-term participation in PReS and PRINTO activities, with significant historic contribution to PR provision of care and research. The centre and disease surveys were presented to all PRINTO centres $(n=288)$ from these 35 countries.

\section{Characteristics of surveys}

The country survey included 10 questions on the healthcare system, organisation of PR care, and access to care; 4 questions on evidence based and qualified care; and 10 questions on education and employment issues (Supplemental Table 1). The Centre survey covered assessment of needs (21 questions), evidence based and qualified care (24 questions), preventive care and adequate 
treatment of co-morbidities (6 questions), transitional care and transferral to adult care (6 questions), and education and employment issues (3 questions; Supplemental Table 2). In 4 disease-specific surveys, 6 questions covered areas of the characteristics and numbers of patients followed in the centre, timing of clinic appointments, use of standardised disease measures, availability of drugs, and patient information strategies.

\section{Data analysis}

Descriptive data were reported as medians $\left(1^{\text {st }}-3^{\text {rd }}\right.$ quartile $)$ for continuous variables; or as absolute numbers and percentages for categorical variables. Percentages were presented for both Eastern and Western European respondents only if they differed by more than $10 \%$.

\section{Results}

From 35 countries approached, 29 representatives replied: 17/18 from Western European Countries (WEC), 12/17 from Eastern European Countries (EEC), with total response rate 83\%. For this analysis, Turkey was grouped with EEC, and Israel with WEC. The Centre survey was returned by 165/288 respondents from 33 countries (125 WEC, 40 EEC; response rate 57\%), ranging between 1 and 21 per country (Figure 1). Upon analysis, results of both surveys were itemised to organisational, quality of care and educational aspects.

\section{Organisation}

\section{General characteristics}

In 25/29 (86\%) countries full coverage is provided for most prescribed medications (94\% WEC, $75 \%$ EEC). Limited coverage of prescribed medication was reported by two EEC only. Inpatient and outpatient medical care is fully covered in $62.5 \%$ (70.6\% WEC, $54.5 \%$ EEC) and $42.9 \%$ of countries, respectively.

\section{Organisation and access to care}

The median number of paediatric rheumatologists per 1 million population was 1.1 (IQR 0.6-1.8 for WEC, 0.5-1.3 for EEC). PR professional organisations were identified in $96.4 \%$ of countries, and combined physicians and allied health professionals (AHP) in $43 \%$ of countries. In $82 \%$ of 
countries such societies organize regular meetings, provide educational materials or provide a platform for communication. In over $60 \%$ of countries, more than half of patients were managed by paediatric rheumatologists in tertiary centres (53\% WEC, $73 \%$ EEC). In $64 \%$ and $46 \%$ of countries a significant minority $(<25 \%)$ of patients were managed by other specialists.

National referral guidelines/clinical recommendations were available in 54\% of countries (59\% WEC, $45 \%$ EEC) as reported by national representatives. Nearly $50 \%$ of responding centres were based in paediatric hospitals (Table 1). From those, 66\% were tertiary centres in university hospitals. More than half of these units acted as paediatric rheumatology national referral centres.

The catchment population for PR services varied significantly according to the size and population of the country and type of centre, ranging from 300000 in Norway to 80 million inhabitants in Germany. Nearly $20 \%$ of patients travelled more than $150 \mathrm{~km}$ to their hospital to obtain access to PR care.

The staff, bed and patient numbers at individual centres are shown in Tables 2 and 3. The average waiting time for new referrals was below 4 weeks in $47.7 \%$ of centres, and was $4-8$ weeks in 21.9\%. A new referral was allocated 30-45 minutes clinical time in 54\% centres. Follow-up visits were 20-30 minutes in 59\% of centres. Combined clinics with other paediatric specialists were available in $78 \%$ of centres.

\section{Quality of care}

Availability of treatments

National guidelines/recommendations for PRD were available in 54\% of countries (59\% WEC, $45 \%$ EEC). Licensed drugs for approved indications were readily available (within weeks) in $96 \%$, without limitation in $43 \%$ (53\% WEC, $27 \%$ EEC), and limited by budget in $29 \%$ of countries (24\% WEC, 36\% EEC).

Disease modifying anti-rheumatic drugs (DMARDs) including subcutaneous methotrexate were readily available in $97 \%$ of centres (100\% WEC, $89 \%$ EEC). Licensed biologics could be prescribed by any qualified paediatric rheumatologist in $82 \%$ of countries ( $88 \%$ WEC, $73 \%$ EEC). 
Limitation of prescription at designated centres was reported by $43 \%$ of countries (35\% WEC, $55 \%$ EEC). Biologics for off-label indications were generally available in 79\% (94\% WEC, 58\% EEC) and could be prescribed without limitations in 21\% (35\% WEC, 0\% EEC), and with budget limitations in $18 \%$ of countries. From $31 \%$ of all individual centres where off-label biologics were not readily available (21\% WEC, $63 \%$ EEC), over $80 \%$ reported regulatory issues as the main barrier to prescription. The use of biologics was recorded in a registry in $96 \%$ of countries: $29 \%$ national, $21 \%$ international, and $29 \%$ both types.

\section{Treatment monitoring, prevention}

Standardised drug monitoring was provided to patients on biologics as well as DMARDs in over $90 \%$ of all centres. For patients on long-term corticosteroids, standardised drug monitoring included regular blood pressure measurement, ophthalmology assessment, and bone densitometry in $90 \%$ of centres ( $88 \%$ WEC, $97 \%$ EEC). Compliance to medication was routinely assessed in $95 \%$ of centres. Treatment outcomes of patient cohorts were regularly reviewed in $72 \%$ of centres (68\% WEC, 86\% EEC).

\section{Transitional care and transferral to adult care}

Specific issues such as alcohol, drugs, sexual health and transition to adult rheumatology were addressed with adolescents in $88 \%$ of centres. Preparation for the transition to adult services started after the age of 16 years in $69 \%$ of centres (65\% WEC, $79 \%$ EEC). Management plans in transfer groups were standardized between paediatric and adult care in $50 \%$ of centres only (53\% WEC, $40 \%$ EEC). Potential difficulties regarding transition to adult care were addressed in the teams of $69 \%$ of centres (75\% WEC, $49 \%$ EEC). Dedicated/transition clinics for adolescent patients were provided by $54 \%$ of centres (58\% WEC, $37 \%$ EEC).

\section{Clinical research}

In over $90 \%$ of centres patients had an opportunity to participate in clinical studies. Laboratorybased scientific research was performed in 41\%; and investigator-initiated clinical research in $70 \%$ of centres (76\% WEC, 51\% EEC). Sponsored drug trials were reported in 54\% of centres. The median number of patients enrolled over the past 5 years per centre were: 100 (IQR 30-200) in 
cohort studies; and 10 (IQR 5-20) in drug trials, similar for WEC and EEC centres. At least part of the team was trained in Good Clinical Practice in $79 \%$ of centres.

\section{Education}

Paediatric rheumatology was officially recognised as a subspecialty in $48.5 \%$ of countries. A syllabus describing the paediatric rheumatic disorders was endorsed by the European Academy of Pediatrics (EAP) and PRES. (8) A defined training scheme for paediatric rheumatology was available in $46 \%$ of countries. Sufficient resources were available for the specialist training in $68 \%$ of countries (59\% WEC, 82\% EEC). Insufficient English language proficiency was identified as an educational burden mainly for AHPs in 5 countries (3 EEC).

Medical schools in $60 \%$ of countries provided under-graduate teaching in PR. A training programme in PR for primary care physicians/general paediatricians was available in $14 \%$ of countries, while a specific training for nurse practitioners in PR was missing in $96 \%$.

\section{Continuing Medical Education (CME)}

Over $80 \%$ of centres reported more than $20 \mathrm{CME}$ hours per year being devoted to PR by their team members. A national medical registration of specific post-graduate/continuing education for physicians existed in $54 \%$ of countries (41\% WEC, $73 \%$ EEC). A similar system for AHP was available only in $18 \%$ of countries. A need for more paediatric rheumatologists was reported by $72 \%$, while a process for manpower planning was available in only $18 \%$ of countries (12\% WEC, $27 \%$ EEC). The majority of countries (93\%) reported an improvement in the situation for PR at the time of this survey as compared to 10 years ago.

\section{Disease-specific issues}

Numbers of patients with individual PRD seen by individual centres are shown in Table 3 . The PRINTO website for families (www.pediatric-rheumatology.printo.it) was the most common patient information resource used by more than $60 \%$ of centres for all groups of PRD. Patientreported outcome tools were available for the assessment of physical functioning (Childhood Health Assessment Questionnaire, CHAQ), quality of life and school attendance. These were 
widely used for all PRD in 37-66\% of all centres. Where available, disease-specific tools were applied by a variable proportion of centres, with JIA assessments being used most widely. There were no significant differences between WEC and EEC centres in the use of any assessment tools.

\section{Discussion}

This is the first pan-European report on the current state-of-art of PR care published to date. In general, PR differs from many other paediatric subspecialties and from adult rheumatology because virtually all PRD are rare diseases. Therefore, direct comparison with other medical specialties, generally caring for both common and rare diseases, is challenging. A comprehensive report from 2011 provided an overview of PR workforce requirements from a US perspective, and identified 4 main educational and economic barriers to service development (9-11). The Author has synthesized published data into specific policy goals offering solutions to each of the barriers. The main attention has been paid to more general aspects of education, organisation, coverage and availability of care. The more recent review describes various approaches of the paediatric rheumatology learning network established in 2011 to improve health outcomes of PRD in USA and Canada(12).

The European PR network has been expanding for more than 20 years. This study aimed to investigate the current organisation and delivery of specialist PR care across Europe and allied countries. Response rates were generally high. A remarkable general finding was the homogeneity of outcomes across replies from 29 countries, despite their significant economic and cultural differences. The PRINTO National Coordinating Centres from the bigger EU member states all replied, as did no less than 165 other paediatric health care providers. That said, the survey was available in English only which may have limited response rate in some countries.

Children with rheumatic conditions should be treated by paediatric rheumatologists. This is in keeping with the replies of 155 centres who indicated that only a minority of children with PRD were treated by other specialists. In the USA almost $25 \%$ of surveyed adult rheumatologists care for children with JIA (13). In Europe most of the paediatric rheumatology care is delivered in tertiary care academic hospitals, especially in EEC (8). 
Availability of national referral guidelines and clinical recommendations for PRD was surprisingly low both in WEC and EEC. This important unmet need is currently being addressed by another work package of the SHARE project, with several sets of recommendations and clinical guidelines now published for various PRD (14-16), as several others pending publication. In 15 countries there were differing replies from individual centres within one country to the question on the existence of an "official system of national referral centres". This may reflect a discrepancy between the clinical practice and official healthcare system rules, or a lack of communication within these country. We also detected limited attention to active patient participation in PR care and research: input from patients and caregivers was collected only in $66 \%$ of centres. Management plans for patient transition from paediatric to adult specialist care were lacking in approximately half of the centres. This is a clear area for improvement that should be endorsed by PReS, and patient advocacy groups in order to better foster patient involvement.

Access to specialist PR clinical services and to standard medications in Europe was good, with nearly $70 \%$ of new patients being seen within 8 weeks of referral. Most prescribed drugs were fully reimbursed in the majority of countries. The availability of expensive biologics for approved indications was surprisingly high, both in the West and the East. Nevertheless, access to their offlabel use was restricted, especially in EEC. Unsurprisingly, over $80 \%$ of centres who reported this problem indicated regulatory issues as the main barrier.

The use of various disease outcome parameters was reported by $37-66 \%$ of centres. Standardised assessment of outcomes is considered one of the main quality improvement methods and has been incorporated among overarching recommendations for the management of PRD $(12,17)$. Disease and treatment registries also belong to clinical research as well as care improvement instruments (18). Accordingly, the majority of patients treated with DMARDs and/or biologics are followed in pharmacovigilance registries. An overall high level of clinical study participation was also encouraging, reflecting the engagement of paediatric rheumatologists and patients alike with rare PRD research.

Although resources for continuing education are higher in WEC, centre replies indicated that national registration of specific post-graduate education exists in only $41 \%$ of WEC, compared to 
$73 \%$ EEC. This finding was relatively independent of the subspecialty recognition status reported by $50 \%$ of both WEC and EEC. To this end, a European Syllabus for Training in Paediatric Rheumatology has been prepared by the PReS Education and Training Committee, and approved by the European Academy of Paediatrics. Also, members of the recently established European Reference Networks (ERN) must document extensive training and registration for their staff (https://ec.europa.eu/health/rare_diseases/european_reference_networks_en). Lack of specialist postgraduate education for allied health professionals is another important unmet need revealed by this study. More detailed analyses of education in PR is a subject of a separate report.

In agreement with reports from other continents (19-21) insufficient numbers of paediatric rheumatologists have been reported by more than $70 \%$ of both EEC and WEC, suggesting that the median number of 1.1 PR specialist physicians per 1 million inhabitants is insufficient. Differences regarding the definition of "paediatric rheumatologist" may account for this discrepancy. For instance, paediatric rheumatologists from smaller countries and centres might devote only $50 \%$ of their clinical hours to this subspecialty service, with the remainder devoted to general paediatric services. The number of specialists thus might not correspond to the number of full-time paediatric rheumatology posts. The suboptimal organisation of care is also reflected by the lack of systematic manpower planning in the subspecialty healthcare in about $80 \%$ of countries.

Our results are limited by the fact that we were not able to verify survey replies by cross-checking individual hospital returns with national healthcare figures, and by the aforementioned limitation that the surveys were only available in English which may have limited responses from some centres. There may be inherent biases, as in all survey studies. For instance, respondents used to higher standards of care could have evaluated more negatively their current resources, whereas responders used to lower standards may have evaluated their services more positively, thus narrowing any potential gaps between East and West. However we do not have any hard evidence to support this notion. Another potential source of bias relates to geographical distribution of centres, which was denser in the West (125 centres) compared to the East (40 centres). This could have biased the results due to inequity of the number of larger high-quality centres in these two geographic locations. Despite that, a strength of our study is the high number of replies from almost all EU countries, and the fact that at the time of final analysis there were no missing answers from 
any of the surveys from the centres that replied. Thus to the best of our ability we believe that the data provide an accurate picture of PR services in the EU and allied countries surveyed.

In conclusion, our survey provides the first comprehensive analysis of current PR specialist services in Europe. We have identified its strengths as well as weaknesses at multiple healthcare levels. Rarity, chronicity and complexity of PRD pose particular challenges for paediatric rheumatology care world-wide. Therefore, understanding what the current state of service provision across the EU is essential in order to further develop optimal care for PRD. Importantly, strengthening existing networks to allow better knowledge transfer regarding best practice, development and dissemination of evidence-based clinical guidelines to benchmark care, and ongoing efforts to facilitate international collaborative research for PRD are key to further improve specialist service provision in paediatric rheumatology across the EU and beyond. Of note, the recent formation of European Reference Networks (ERN) on rare diseases within the EU project has been an important starting point for healthcare improvement in this area.

\section{Acknowledgement:}

SHARE, grant number No. 20111202

all the country representatives who replied (national coordinators) Printo to provide the list please 
Table 1: Type and size of hospitals/units where paediatric rheumatology centres are based

\begin{tabular}{|l|c|c|c|}
\hline Centre origin & WEC & EEC & Total \\
\hline \hline $\mathbf{N}$ & 125 & 40 & 165 \\
\hline \hline Children's hospital & $54(43.2 \%)$ & $23(57.5 \%)$ & $77(46.7 \%)$ \\
\hline General hospital & $35(28.0 \%)$ & $4(10.0 \%)$ & $39(23.6 \%)$ \\
\hline Both & $29(23.2 \%)$ & $9(22.5 \%)$ & $38(23.0 \%)$ \\
\hline \hline Other (specify) & $7(5.6 \%)$ & $4(10.0 \%)$ & $11(6.7 \%)$ \\
\hline $\begin{array}{l}\text { Number of all paediatric inpatient } \\
\text { beds }\end{array}$ & $110(40-175)$ & $112(60-233)$ & $111(40-195)$ \\
\hline $\begin{array}{l}\text { Number of paediatric } \\
\text { rheumatology inpatient beds }\end{array}$ & $3(1-5)$ & $8(5-13)$ & $4(2-8)$ \\
\hline $\begin{array}{l}\text { Number of all paediatric } \\
\text { outpatients per week }\end{array}$ & $350(150-775)$ & $400(135-1033)$ & $400(150.0-889)$ \\
\hline $\begin{array}{l}\text { Number of paediatric } \\
\text { rheumatology outpatients per } \\
\text { week }\end{array}$ & $30(15-50)$ & $48(25-85)$ & $30(20-50)$ \\
\hline
\end{tabular}

Legend: WEC Western European Countries EEC Eastern European Countries

Table 2: Paediatric Rheumatology Centre characteristics

\begin{tabular}{|l|l|l|}
\hline & Median Number & $1^{\text {st }} 3^{\text {rd }}$ quartile \\
\hline Paediatric Rheumatologists & 3 & $2-4$ \\
\hline Fellows/Trainees & 1 & $1-2$ \\
\hline Nurses & 2 & $1-4$ \\
\hline Other AHP & 2 & $1-3$ \\
\hline Paediatric Beds total & 111 & $40-211$ \\
\hline Paediatric Rheumatology beds & 4 & $2-8$ \\
\hline $\begin{array}{l}\text { Paediatric Outpatients } \\
\text { total/week }\end{array}$ & 425 & $150-897$ \\
\hline $\begin{array}{l}\text { Paediatric Rheumatology } \\
\text { Outpatients/week }\end{array}$ & 30 & $20-52$ \\
\hline
\end{tabular}

Legend: AHP Allied Health Professionals 
Table 3: Patient population characteristics in individual centres: Median numbers of reviewed and new patients in one year per centre

\begin{tabular}{|l|c|c|c|}
\hline & 125 WES & 40 EEC & 165 Total \\
\hline \hline & Total / New & Total / new & Total / new \\
\hline JIA & $165 / 20$ & $181 / 30$ & $168 / 25$ \\
\hline SLE & $8 / 2$ & $10 / 3$ & $9 / 2$ \\
\hline \hline APS & $1 / 0$ & $2 / 1$ & $1 / 0$ \\
\hline VAS & $4 / 1$ & $6 / 2$ & $5 / 1$ \\
\hline JIIM & $5 / 1$ & $5 / 2$ & $5 / 1$ \\
\hline JSS & $0 / 0$ & $1 / 1$ & $1 / 0$ \\
\hline AID & $15 / 3$ & $12 / 5$ & $14 / 4$ \\
\hline \hline
\end{tabular}

Legend: WEC Western European Countries EEC Eastern European Countries JIA Juvenile Idiopathic Arthritis JIIM Juvenile Idiopathic Inflammatory Myopathies JS Juvenile Scleroderma SLE Systemic Lupus Erythematosus APS Antiphospholipid syndrome VAS Vasculitides AID Autoinflammatory Diseases 


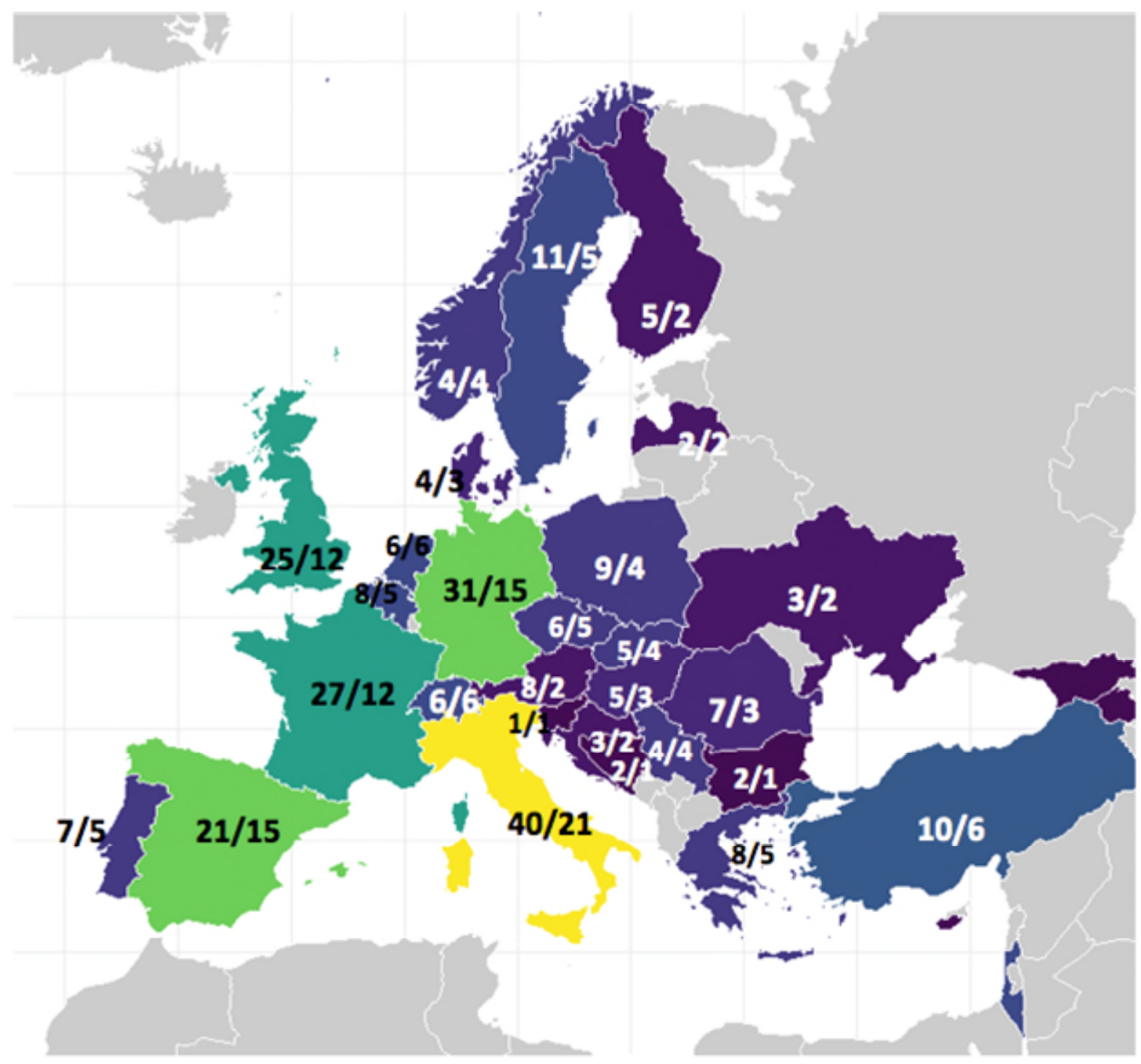

N 
Figure 1: Overview of countries participating in the survey. The numbers indicate the ratio of the total number of sites in the country, and the number of sites that actually participated

\section{Supplemental material}

Table 1: Country survey

Table 2: Centre survey

\section{References}

1. Wulffraat NM, Vastert B, consortium S. Time to share. Pediatr Rheumatol Online J. 2013;11(1):5.

2. Consolaro A, Ruperto N, Bazso A, Pistorio A, Magni-Manzoni S, Filocamo G, et al. Development and validation of a composite disease activity score for juvenile idiopathic arthritis. Arthritis Rheum. 2009;61(5):658-66.

3. Consolaro A, Ruperto N, Bracciolini G, Frisina A, Gallo MC, Pistorio A, et al. Defining criteria for high disease activity in juvenile idiopathic arthritis based on the juvenile arthritis disease activity score. Ann Rheum Dis. 2014;73(7):1380-3.

4. Ruperto N, Brunner HI, Quartier P, Constantin T, Wulffraat N, Horneff G, et al. Two randomized trials of canakinumab in systemic juvenile idiopathic arthritis. N Engl J Med. 2012;367(25):2396-406.

5. Ruperto N, Garcia-Munitis P, Villa L, Pesce M, Aggarwal A, Fasth A, et al. PRINTO/PRES international website for families of children with rheumatic diseases: www.pediatric-rheumatology.printo.it. Ann Rheum Dis. 2005;64(7):1101-6.

6. Ruperto N, Ravelli A, Cuttica R, Espada G, Ozen S, Porras O, et al. The Pediatric Rheumatology International Trials Organization criteria for the evaluation of response to therapy in juvenile systemic lupus erythematosus: prospective validation of the disease activity core set. Arthritis Rheum. 2005;52(9):2854-64.

7. Wulffraat N, van der Net JJ, Ruperto N, Kamphuis S, Prakken BJ, Ten Cate R, et al. The Dutch version of the Childhood Health Assessment Questionnaire (CHAQ) and the Child Health Questionnaire (CHQ). Clin Exp Rheumatol. 2001;19(4 Suppl 23):S111-5.

8. Avčin TA, J.; Cimaz, R.; Foster, H.E.; Harjaček, M.; Hofer, M.; Martini, A.; Smith, E.; Uziel, Y.; Net, van der JJ.; Wouters, C.; Wulffraat, N.M.;. European Syllabus for Training in Paediatric Rheumatology 2016. On behalf of European Academy of Pediatrics and the European Board of Pediatrics [Available from: www.pres.eu/committee-and-wp/education.html.

9. Henrickson M. Policy challenges for the pediatric rheumatology workforce: Part III. the international situation. Pediatr Rheumatol Online J. 2011;9:26.

10. Henrickson M. Policy challenges for the pediatric rheumatology workforce: Part I. Education and economics. Pediatr Rheumatol Online J. 2011;9:23.

11. Henrickson M. Policy challenges for the pediatric rheumatology workforce: Part II. Health care system delivery and workforce supply. Pediatr Rheumatol Online J. 2011;9:24. 
12. Harris JG, Bingham CA, Morgan EM. Improving care delivery and outcomes in pediatric rheumatic diseases. Curr Opin Rheumatol. 2016;28(2):110-6.

13. Van Mater H, Balevic SJ, Freed GL, Clark SJ. Prescribing for Children with

Rheumatologic Disease: Perceived Treatment Approaches between Pediatric and Adult Rheumatologists. Arthritis Care Res (Hoboken). 2017.

14. Giancane G, Ter Haar NM, Wulffraat N, Vastert SJ, Barron K, Hentgen V, et al. Evidence-based recommendations for genetic diagnosis of familial Mediterranean fever. Ann Rheum Dis. 2015;74(4):635-41.

15. Enders FB, Bader-Meunier B, Baildam E, Constantin T, Dolezalova P, Feldman BM, et al. Consensus-based recommendations for the management of juvenile dermatomyositis. Ann Rheum Dis. 2017;76(2):329-40.

16. ter Haar NM, Oswald M, Jeyaratnam J, Anton J, Barron KS, Brogan PA, et al. Recommendations for the management of autoinflammatory diseases. Ann Rheum Dis. 2015;74(9):1636-44.

17. Swart JF, van Dijkhuizen EHP, Wulffraat NM, de Roock S. Clinical Juvenile Arthritis Disease Activity Score proves to be a useful tool in treat-to-target therapy in juvenile idiopathic arthritis. Ann Rheum Dis. 2017.

18. Gliklich RE, Leavy MB, Velentgas P, Dreyer NA, Tunis SR, Mohr P, et al. Incorporating stakeholder perspectives in developing a translation table framework for comparative effectiveness research. J Comp Eff Res. 2012;1(3):281-92.

19. Barber CE, Jewett L, Badley EM, Lacaille D, Cividino A, Ahluwalia V, et al. Stand Up and Be Counted: Measuring and Mapping the Rheumatology Workforce in Canada. J Rheumatol. 2017;44(2):248-57.

20. Cox A, Piper S, Singh-Grewal D. Pediatric rheumatology consultant workforce in Australia and New Zealand: the current state of play and challenges for the future. Int J Rheum Dis. 2017;20(5):647-53.

21. Reveille JD, Munoz R, Soriano E, Albanese M, Espada G, Lozada CJ, et al. Review of Current Workforce for Rheumatology in the Countries of the Americas 2012-2015. J Clin Rheumatol. 2016;22(8):405-10. 